\title{
Production of Dark Matter in Warped Higgsless Models with Composite Sector Supersymmetry
}

\author{
Alexander Knochel ${ }^{* a, b}$ \\ Thorsten $\mathrm{Ohl}^{\dagger a}$ \\ ${ }^{a}$ Institut für Theoretische Physik und Astrophysik \\ Universität Würzburg \\ Am Hubland, 97074 Würzburg, Germany \\ ${ }^{b}$ Albert-Ludwigs Universität Freiburg \\ Institut für Physik \\ Hermann-Herder-Str. 3, 79104 Freiburg, Germany
}

October 15,2018

\begin{abstract}
We explore the LHC phenomenology of an extension of warped higgsless models. The model is supersymmetric in the bulk and on the IR brane as introduced in [1, corresponding to an emergence of a supersymmetric spectrum in the composite sector of the higgsless model. In particular, the lightest neutralino is rendered stable by an $R$ parity and serves as a realistic cold dark matter candidate. The observation of missing energy signals at the LHC from LSP and NLSP production in association with third generation quarks is discussed based on simulations using WHIZARD.
\end{abstract}

*e-mail: aknochel@physik. uni-wuerzburg.de

${ }^{\dagger}$ e-mail: ohl@physik. uni-wuerzburg.de 


\section{Introduction}

Two of the most prominent issues to be addressed at the Large Hadron Collider (LHC) are the origin of electroweak symmetry breaking (EWSB) and the nature of dark matter (DM) which, according to recent results of the WMAP experiment, makes up roughly $20 \%$ of the energy density of the observable universe. The Standard Model (SM) of particle physics, when extended to include massive neutrinos, is extremely successful at describing the observations made at past and present experiments, including many nontrivial precision tests. Despite these achievements, it fails to provide a candidate for cold DM in the form of a stable, weakly interacting particle species, but at the same time predicts a scalar Higgs particle which so far has eluded detection at the LEP and Tevatron experiments. In addition, the minimal Higgs mechanism is theoretically unappealing due to the severe fine tuning required by quadratically divergent radiative corrections to the Higgs potential. It is probably not a coincidence that all of the problems mentioned above, the observed DM relic density $\Omega h^{2} \approx 0.1$ and the shortcomings of the standard EWSB scenario, strongly point to a modification and/or extension of the SM at the $\mathrm{TeV}$ scale which is thus within reach of the LHC experiments. We take the apparent absence of the SM Higgs boson below $\approx 115$ $\mathrm{GeV}$ and the cosmological evidence for weakly interacting stable particles at or below the $\mathrm{TeV}$ scale as a motivation to consider an alternative scenario which relates both issues, namely a supersymmetric formulation of higgsless models in a warped extra dimension in which the properties of the gaugino dark matter candidate are closely tied to the mechanism which produces the electroweak gauge boson masses. We have introduced the model setup in [1] including a discussion of the virtues of the lightest neutralino modes as a cold dark matter (CDM) candidate.

This paper is organized as follows: In section 2 we give a short review of the model including the superfield content, the boundary conditions imposed to obtain a realistic light spectrum, and some key properties. We discuss the parameters which have an important impact on LSP phenomenology at the LHC, and define a set of benchmark points in parameter space. In section 3 we discuss promising channels for an observation of missing energy from LSP pair production. Our conclusions are based on the simulation of four parton final states at the LHC with WHIZARD [2]. We propose sets of kinematical cuts and present the corresponding results for a range of model parameters. In section 4 we summarize our results. 


\section{The Model}

\subsection{General Setup}

The model [1] on which we base the following discussions is an extension of the warped higgsless model proposed in [3]. The 5D gauge group is

$$
G_{b u l k}=S U(3)_{C} \times S U(2)_{L} \times S U(2)_{R} \times U(1)_{B-L},
$$

with the corresponding $5 \mathrm{D}$ gauge coupling constants $g_{5 S}, g_{5 L}=g_{5 R}=g_{5}$ and $\tilde{g}_{5}$. The full symmetry is conserved in the bulk and broken by boundary conditions to

$$
G_{\text {bulk }} \rightarrow\left\{\begin{array}{cl}
G_{S M}=S U(3)_{C} \times S U(2)_{L} \times U(1)_{Y} & \text { on the UV brane } \\
S U(3)_{C} \times S U(2)_{D} \times U(1)_{B-L} & \text { on the IR brane }
\end{array}\right.
$$

and thus only $S U(3)_{C} \times U(1)_{E M}$ survives as an unbroken subgroup. Matter hypermultiplet masses are generated on the IR brane as usual where they are allowed by the $S U(2)_{D}$ symmetry, while the isospin doublets are split by Majorana masses and localized kinetic terms on the UV brane where $S U(2)_{R}$ is broken.

Since the smallest spinors in 5D are of Dirac type, there are four supercharges in $5 \mathrm{D} \mathcal{N}=1 \mathrm{SUSY}$, which can be related to $4 \mathrm{D} \mathcal{N}=2$ SUSY. However, half of the symmetries are broken by the background, leaving us with usual $\mathcal{N}=1$ SUSY after Kaluza-Klein expansion. Nevertheless, we assume that full 5D hypermultiplets with corresponding interactions are present in the bulk of the extra dimension in such a way that full 5D SUSY spectra appear at energies $E>\Lambda_{I R}$. The corresponding action of hypermultiplets coupled to 5D Super Yang-Mills theory broken by a warped background can be expressed by ordinary $\mathcal{N}=1$ chiral- and vector superfields [4]. The resulting superfield content with representations and quantum numbers is summarized in table 1. Each hypermultiplet has a bulk mass

$$
M_{i}=c_{i} k,
$$

parameterized in units of the Randall-Sundrum curvature $k$ which acts as a localization parameter for the entire multiplet.

To define the full model, one needs to specify the boundary conditions and brane action. The IR brane (i. e. $y=\pi$ ) boundary conditions are a straightforward generalization of the nonsupersymmetric boundary conditions [1]. In contrast, SUSY is broken on the UV brane, which means that boundary conditions can differ within 4D multiplets. We assume a localized kinetic 


\begin{tabular}{llll} 
Field & Representations & Field & Representations \\
\hline$V^{C a}, \chi^{C a}$ & $S U(3)_{C}$ gauge multiplet & $V^{L i}, \chi^{L i}$ & $S U(2)_{L}$ gauge multiplet \\
$V^{R i}, \chi^{R i}$ & $S U(2)_{R}$ gauge multiplet & $V^{X}, \chi^{X}$ & $U(1)_{B-L}$ gauge multiplet \\
$H_{l, g}^{L}$ & $(\mathbf{1}, \mathbf{2}, \mathbf{1})_{-1}$ & $H_{l, g}^{R}$ & $(\mathbf{1}, \mathbf{1}, \mathbf{2})_{-1}$ \\
$H_{l, g}^{L c}$ & $(\mathbf{1}, \overline{\mathbf{2}}, \mathbf{1})_{1}$ & $H_{l, g}^{R c}$ & $(\mathbf{1}, \mathbf{1}, \overline{\mathbf{2}})_{1}$ \\
$H_{q, g}^{L}$ & $(\mathbf{3}, \mathbf{2}, \mathbf{1})_{1 / 3}$ & $H_{q, g}^{R}$ & $(\mathbf{3}, \mathbf{1}, \mathbf{2})_{1 / 3}$ \\
$H_{q, g}^{L c}$ & $(\overline{\mathbf{3}}, \overline{\mathbf{2}}, \mathbf{1})_{-1 / 3}$ & $H_{q, g}^{R c}$ & $(\overline{\mathbf{3}}, \mathbf{1}, \overline{\mathbf{2}})_{-1 / 3}$
\end{tabular}

Table 1: The superfield content of the model and corresponding representations and quantum numbers with respect to the bulk gauge group $G_{\text {bulk }}=S U(3)_{C} \times S U(2)_{L} \times S U(2)_{R} \times U(1)_{B-L}$. Here, $V$ denotes vector superfields, $\chi$ denotes chiral superfields in the adjoint as they appear in $\mathcal{N}=2$ vector multiplets, and $H$ the denotes the chiral superfield component of the matter hypermultiplets. $g=1 \ldots 3$ denotes the generation.

term for the $S U(2)_{R}$ transforming fields on the UV brane controlled by a parameter $\rho$, which yields effective boundary conditions after Kaluza-Klein expansion

$$
f_{q}^{R}(0)=m \rho^{2} f_{q^{c}}^{R}(0),
$$

while neutrinos can receive a UV localized Majorana mass $\mu_{r}$, leading to effective boundary conditions,

$$
\psi_{\nu}^{R}(0)=\frac{\mu_{r}}{k} \psi_{\nu}^{R c}(0)
$$

The physical scalars $h_{f}, h_{f}^{c}, \Sigma^{i}$ get universal Dirichlet conditions

$$
h_{f}(0)=h_{f}^{c}(0)=\Sigma^{i}(0)=0
$$

and the gluinos receive boundary conditions that are "twisted" with respect to the gluons

$$
\lambda_{1}^{C}(0)=0 .
$$

For the electroweak gauginos there are several possibilities. The neutral sector consists of three $5 \mathrm{D}$ gauginos, $\lambda_{i}^{L 3}, \lambda_{i}^{R 3}, \lambda_{i}^{X}$, while the charged sector is spanned by $\lambda_{i}^{L \pm}$ and $\lambda_{i}^{R \pm}$. The boundary conditions of the charged and neutral sectors are linked by the $S U(2)_{L}$ symmetry. The neutralino boundary conditions can be classified by the number of twisted/untwisted conditions imposed. Furthermore, the fields $\lambda^{X}$ and $\lambda^{R 3}$ can mix on the UV brane. 
However, only two choices remove all massless gaugino modes automatically from the spectrum, both with two twisted boundary conditions in the neutral sector. The first one is

$$
\lambda_{2}^{L}=\lambda_{1}^{R}=\lambda_{1}^{X}=0
$$

while in the second

$$
\begin{aligned}
\lambda_{1}^{L} & =\lambda_{2}^{R 12}=0 \\
\cos \left(\theta_{N}\right) \lambda_{1}^{X}+\sin \left(\theta_{N}\right) \lambda_{1}^{R 3} & =\cos \left(\theta_{N}\right) \lambda_{2}^{R 3}-\sin \left(\theta_{N}\right) \lambda_{2}^{X}=0 .
\end{aligned}
$$

Since in the former, charginos and neutralinos are degenerate with the $W$ bosons and neutralino annihilation cross sections are too large, we choose the latter [1]. This completes the list of boundary conditions.

\subsection{Enhanced Cross Sections from Almost Delocalized Quarks}

While the parameter space of the model is still rather large, there are several reasonable assumptions that one can make. First of all, at tree level, we impose degeneracy of the pairs of electroweak gaugino modes which will be lifted only at the loop level (i. e. no Majorana masses on the UV brane). Furthermore, the splitting of the $W$ and $\chi^{+}$raises the KK scale and is therefore assumed to be as small as possible in order to achieve unitarization (i. e. with the lightest charginos just above the experimental exclusion range). The neutralino mixing angle $\theta_{N}$ is then fixed by the relic density. The localization of the light quarks is largely constrained by the $S$ parameter to be close to $c_{L}=0.5$, which also suppresses the coupling to the heavy resonances. The third generation is naturally IR localized to generate the heavy top.

While this basically fixes the properties that are relevant to the LSP production processes which we will discuss later, there remains some freedom in the localization parameters which strongly impact LHC phenomenology. Let us therefore discuss them in more detail. Exactly delocalized light quarks $\left(c_{L}=-c_{R}=1 / 2\right)$ have vanishing couplings to KK gluons, while a small deviation from delocalization introduces nonzero couplings. At the same time, localized kinetic terms for the quarks which are used to split the isospin doublets, introduce a localization effect on the UV brane which also shifts these effective couplings to a nonzero value.

The importance of KK gluon intermediate states in our study of LSP production at the LHC depends on the exact choices of localization parameters. Our minimal implementation of the third generation, though not addressing the $Z b b$ problem [5], provides a simple way to study the phenomenology of the $t$ and $b$ in LSP production for different scenarios from strongly IR 


\begin{tabular}{c|c|c|c|c|c|c} 
Bulk Mass & P1 & P2 & P3 & P4 & P5 & P6 \\
\hline \hline$c_{L 1,2}$ & 0.48 & 0.48 & $1 / 2$ & 0.48 & 0.48 & 0.48 \\
\hline$c_{R 1,2}$ & -0.48 & -0.48 & $-1 / 2$ & -0.48 & -0.48 & -0.48 \\
\hline$c_{L 3}$ & $1 / 3$ & 0.4 & 0.4 & 0.2 & 0.2 & 0.4 \\
\hline$c_{R 3}$ & -0.4 & $-1 / 3$ & $-1 / 3$ & -0.2 & -0.4 & -0.2
\end{tabular}

Table 2: Definitions of points in bulk mass parameter space of the first and second $\left(c_{1,2}\right)$ and third $\left(c_{3}\right)$ generation of quarks.

localized fields to the almost delocalized case. The coupling strength of a (righthanded) quark zero mode

$$
f_{\psi}=N z^{2+c_{R}}
$$

to the KK gluon,

$$
\frac{g_{s}^{\prime}}{g_{s} \sqrt{\pi R}}=\left\langle f_{R} f_{R} f_{g}^{\prime}\right\rangle=\frac{\int\left[(z k)^{-4}+\rho_{q}^{2} \delta\left(z-k^{-1}\right)\right] d z z^{4+2 c_{R}} f_{g}^{\prime}}{\rho_{q}^{2} k^{-2 c_{R}-4}+\int(z k)^{-4} d z z^{4+2 c_{R}}}
$$

is shown in figure 1 as a function of the bulk mass $c_{L}=-c_{R}$. As expected, the introduction of a UV localized kinetic term for the quarks shifts the effective localization. While the localization of the third generation lets the mass of the first quark KK modes vary between extremely light (for almost delocalized third generation fields requiring large IR Dirac masses) and heavy $\left(\approx 3 k e^{-R k \pi}\right)$, the masses of the lightest $\tilde{t}$ and $\tilde{b}$ modes stay below $2 k e^{-R k \pi} \approx$ $1100 \mathrm{GeV}$ and can thus be pair produced at LHC energies with appreciable cross sections. We thus define in table 2 five points in parameter space which represent extreme and intermediate cases of quark localization,

The points P1 and P2 feature slightly IR localized light quarks corresponding to vanishing tree level contributions to $S$, while having small but nonzero couplings to KK gluons. In $\mathrm{P} 1$, the bulk masses of $S U(2)_{L}$ and $S U(2)_{R}$ transforming hypermultiplets are exchanged relative to P2. P3 is a variation of P2 with exactly delocalized light lefthanded quarks. P4 features rather strongly IR localized third generation hypermultiplets, while the points P5 and P6 correspond to a more asymmetric third generation localization.

\subsection{The Particle Spectrum}

The spectrum of new particles in the LHC range is given by the KK resonances and their $\mathcal{N}=2$ superpartners residing at $600 \ldots 1500 \mathrm{GeV}$, some 


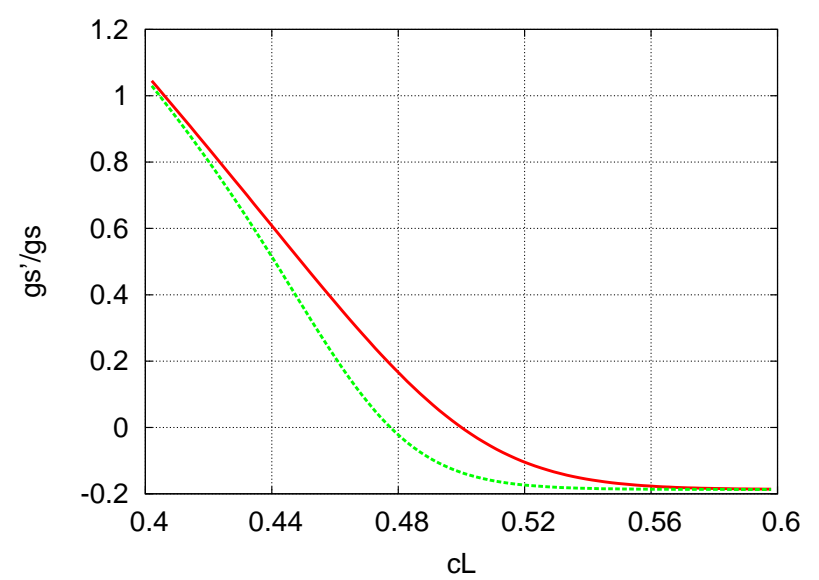

Figure 1: The coupling of a massless quark to the first gluon resonance as a function of the bulk mass. The upper line is for $\rho=0$, while the lower one is for $\rho^{2} k=100$, which corresponds roughly to the $u-d$ splitting at $c_{L}=-c_{R}=1 / 2$.

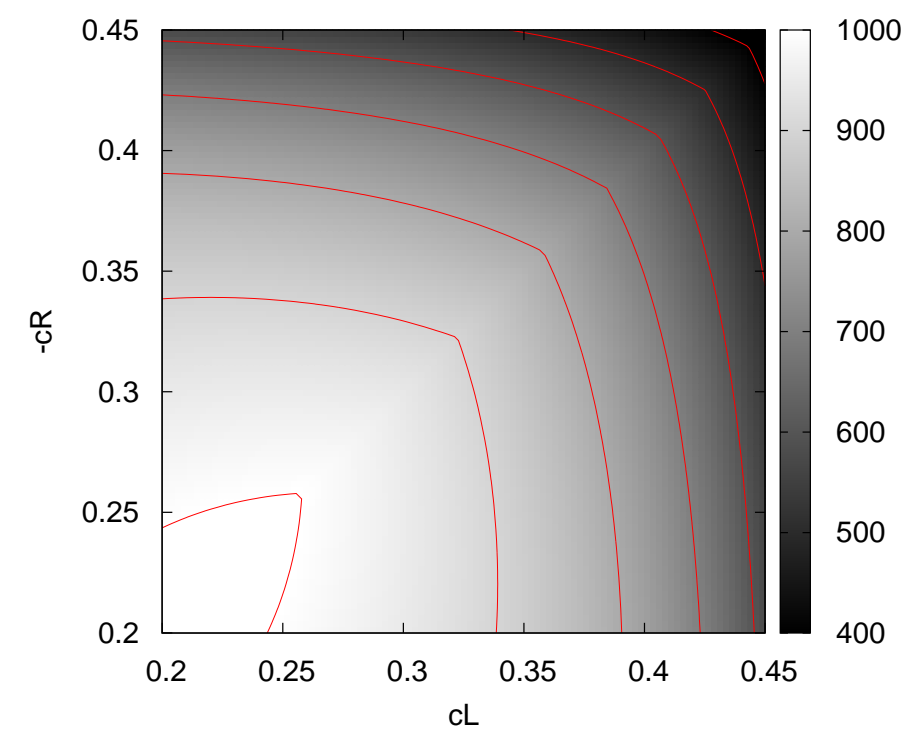

Figure 2: The mass of the lightest stop resonance in $\mathrm{GeV}$ as a function of the third generation bulk masses for $\Lambda_{I R} \approx 600 \mathrm{GeV}$. 
of which are very broad (e.g. the color charged resonances $\tilde{g}, g^{\prime}$ ), while the $f^{\prime}, \tilde{f}$ can be comparatively narrow with $\Gamma<100 \mathrm{GeV}$. Due to boundary conditions, there are no sfermion or gluino zero modes, making otherwise unprotected UV localized light scalars unnatural. The relevant two body decay modes of the light $\tilde{q}$ are into $\chi^{ \pm 0} q$. However, as soon as any resonance is heavy enough to allow KK fermions or KK gauginos in the final state those dominate due to the strongly enhanced couplings. The first KK fermions have strong electroweak two body decays $f \rightarrow W / Z, f$, while the KK charginos and neutralinos themselves mainly decay into $t \tilde{t}, b \tilde{b}$, gluinos in addition to $t^{\prime} \tilde{t}, b^{\prime} \tilde{b}$, both with enhanced couplings due to IR localization of the third generation. For the same reason, the gluon decays to $t \bar{t}, t^{\prime} \bar{t}$ etc. with total widths varying between $\approx 300 \ldots 500 \mathrm{GeV}$, while the scalar gluon coupling is very sensitive to quark localization and vanishes in the LR symmetric case, resulting in widths ranging from $\Gamma \approx 10 \ldots 100 \mathrm{GeV}$ in which the $t^{\prime} \bar{t}$ final state dominates. The masses and widths of the relevant color charged resonances are summarized in table 3 .

\section{Production of the LSP}

\subsection{Heavy Quarks and Missing Energy at the LHC}

Stable neutralinos (and very likely any other DM candidates which are not yet ruled out by direct searches) are invisible to the detectors at collider experiments. This means that, even though our LSP might be produced copiously in processes of the type $q \bar{q} \rightarrow \chi^{0} \chi^{0}$, the remainders of the protons go down the beam line, and since we lack information about the total cross section, the process is entirely invisible. Also, we can not use the recoil spectrum in $q \bar{q} \rightarrow \chi^{0} \chi^{0} j$ because the longitudinal momenta of partons are not known.

The most promising approach is therefore to observe the associated production with other easily detectable objects such as leptons or hard jets, and to look for missing momentum in the direction transverse to the proton beams, $P_{T}$, which experiments are equipped to measure by balancing the visible momentum. One such process which we will however not pursue further is the electroweak production channel of neutralinos and charginos by VBF. Instead we concentrate on another set of final states which is particularly favored in the model which we consider in this work, the production of third generation quarks in association with missing energy. In general, it can be an important process for discovering scenarios of WIMP DM. It is particularly interesting when a "partner" of heavy quarks exists, which decays directly to 


\begin{tabular}{c|cccccc} 
Pt. & P2 & P3 & P1 & P4 & P5 & P6 \\
\hline$t^{1}$ & $970(30)$ & $970(30)$ & $970(40)$ & $1480(90)$ & $1180(60)$ & $1180(60)$ \\
\hline$t^{2}$ & $2140(460)$ & $2140(460)$ & $2140(480)$ & $2050(100)$ & $2120(340)$ & $2120(300)$ \\
\hline$\tilde{t}^{1}$ & $880(40)$ & $880(40)$ & $800(20)$ & $1100(40)$ & $860(20)$ & $1080(110)$ \\
\hline$\tilde{t}^{1 c}$ & $800(80)$ & $800(80)$ & $880(130)$ & $1100(140)$ & $1080(230)$ & $860(80)$ \\
\hline$\tilde{t}^{2}$ & $2040(450)$ & $2040(450)$ & $1970(320)$ & $1850(180)$ & $1840(120)$ & $2020(420)$ \\
\hline$\tilde{t}^{c 2}$ & $1970(350)$ & $1970(350)$ & $2040(490)$ & $1850(340)$ & $2020(490)$ & $1840(160)$
\end{tabular}

\begin{tabular}{c|cccccc} 
Pt. & P2 & P3 & P1 & P4 & P5 & P6 \\
\hline$b^{1}$ & $800(40)$ & $800(40)$ & $880(50)$ & $1100(60)$ & $1080(80)$ & $860(50)$ \\
\hline$b^{2}$ & $1970(470)$ & $1970(470)$ & $2040(480)$ & $1850(150)$ & $2020(340)$ & $1840(320)$ \\
\hline$\tilde{b}^{1}$ & $880(80)$ & $880(80)$ & $800(40)$ & $1100(80)$ & $860(40)$ & $1080(160)$ \\
\hline$\tilde{b}^{c 1}$ & $800(70)$ & $800(70)$ & $880(120)$ & $1100(130)$ & $1080(220)$ & $860(70)$ \\
\hline$\tilde{b}^{2}$ & $2040(280)$ & $2040(280)$ & $1970(270)$ & $1850(90)$ & $1840(110)$ & $2020(170)$ \\
\hline$\tilde{b}^{c 2}$ & $1970(380)$ & $1970(380)$ & $2040(540)$ & $1850(340)$ & $2020(560)$ & $1840(190)$
\end{tabular}

\begin{tabular}{c|cccccc} 
Pt. & P2 & P3 & P1 & P4 & P5 & P6 \\
\hline$g^{\prime}$ & $1520(330)$ & $1520(330)$ & $1520(390)$ & $1520(420)$ & $1520(430)$ & $1520(290)$ \\
\hline$\Sigma_{C}$ & $1520(100)$ & $1520(100)$ & $1520(100)$ & $1520(10)$ & $1520(50)$ & $1520(50)$ \\
\hline$\tilde{g}$ & $1500(290)$ & $1500(290)$ & $1500(360)$ & $1500(210)$ & $1500(380)$ & $1500(230)$
\end{tabular}

Table 3: Masses (and widths) in $\mathrm{GeV}$ of the lowest color charged resonances at tree level in the third generation hypermultiplets and $\mathrm{SU}(3)$ gauge multiplets. Only decays to two parton final states are considered here. 
a heavy quark (preferably the top) and invisible particles such as in SUSY with $\mathrm{R}$ parity. In our scenario, these partners are the first stop modes $\tilde{t}$ and they are in a convenient mass range: still light enough to be pair produced copiously at the LHC at $14 \mathrm{TeV}$, and at the same time heavy enough $\left(m_{\tilde{t}}-m_{\chi}-m_{t} \gtrsim 400 \mathrm{GeV}\right)$ in all but the extreme cases to produce large missing energy contributions from the decay. Such a situation has been discussed in a generic way in [7]. The analysis carried out by the authors is valid for our $\tilde{t}$ pair production contributions, but this is only one of the contributions to this class of final states in our model, where the production of heavy quark and gluon resonances proves to be important as well. Due to the large number of interaction vertices in the model and the corresponding number of Feynman diagrams, we simulate only four parton final states, which means that we do not consider the possible decay modes of the $t$. When judging the results for the missing energy signal with $t$ and $b$ quarks in the final state, one therefore has to remember the following points: the $t$ pair production itself does not introduce $\not_{T}$, but the leptonic and semileptonic decay modes contain neutrinos, and considering the relative strength of $t$ pair production, this can constitute an important background. In addition to this, there are SM processes with have final states distinguishable from our signal only by their kinematics, for example $p p \rightarrow b \bar{b} \nu l j j$ [7] or $p p \rightarrow b \bar{b} \nu l \nu l$ corresponding to semileptonic and leptonic decays of top pair production. While a complete analysis of these backgrounds is beyond the scope of this work, there is a generic way to suppress them: as will become apparent in the following discussion, we can afford to place rather strong $P_{T}$ and $\Delta \phi(q, \bar{q})$ cuts without losing too much of our signal - this strategy would be futile if there was only a small mass gap between the $t$, neutralinos and the heavy partners. We demonstrate this for the leading SM background from leptonic top decays.

Let us have a closer look at the model to see how the production of heavy quarks is suited for the discovery of our DM candidate via $\not_{T}$. The couplings of the neutralino to light matter are of electroweak strength, and considering the vertices in the model, the only production process at $\mathcal{O}\left(\alpha_{s} \alpha\right)$ is through squarks. However, even though these are Kaluza-Klein in our version of UV brane symmetry breaking, they can be produced on shell and decay through

$$
\tilde{q} \longrightarrow q \chi^{0 \pm}
$$

due to the heavyness of the gluino, making this effectively an $\mathcal{O}\left(\alpha_{s}\right)$ process which is however suppressed by the large parton momentum fraction $x$ needed to produce two onshell squarks. We can also potentially obtain final states with two quarks and two LSPs by producing a heavy chargino or neutralino 

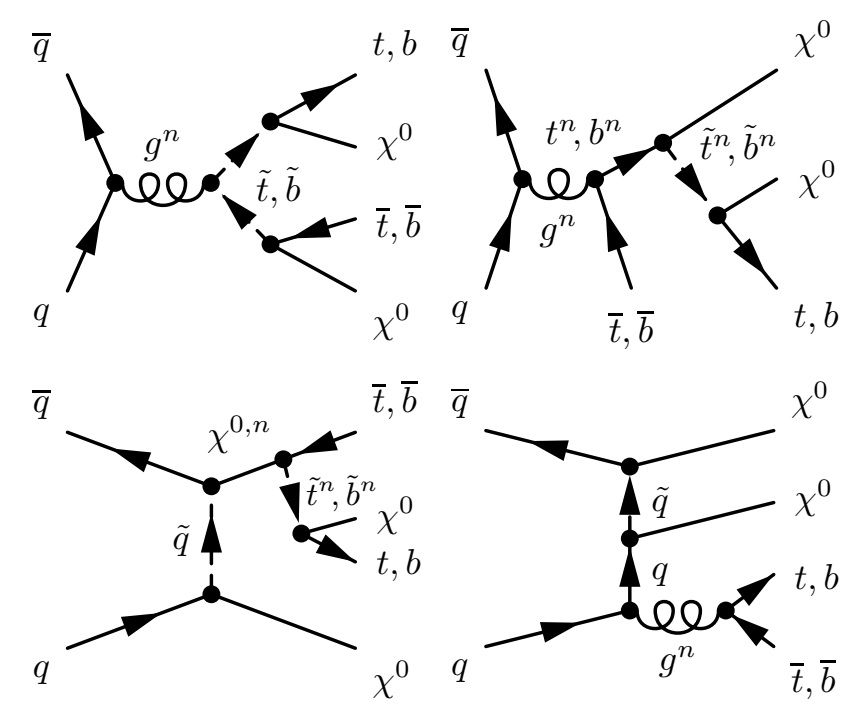

Figure 3: The relevant tree level contributions (up to charge conjugation and crossing) to associated heavy quark and LSP pair production with a $q \bar{q}$ initial state.

resonance which then decays to the LSP and two quarks through the decay chain shown in figure 3 ,

$$
\begin{aligned}
q \bar{q} \rightarrow \chi^{0} \chi^{0 \pm, 2 / 3}, & \chi^{0 \pm, 2 / 3} \rightarrow \tilde{q} \bar{q} \rightarrow \chi^{0} q \bar{q}+c . c \\
q \bar{q} \rightarrow \chi^{0} \tilde{g}, & \tilde{g} \rightarrow \tilde{q} \bar{q} \rightarrow \chi^{0} q \bar{q}+c . c .
\end{aligned}
$$

but these channels are suppressed because of the small coupling of a light neutralino to squarks and light quarks and, like for LSP annihilation, they do not play a significant role. Kaluza-Klein top production and decay (figure 3) is not as suppressed because of the IR localization (and wavefunction deformation from IR masses) of the top quark, and we can thus have

$$
q \bar{q} \rightarrow \bar{t} t^{2} \quad t^{2} \rightarrow \chi^{0} \tilde{t}^{1} \rightarrow \chi^{0} \chi^{0} t
$$

as unique new contributions in addition to pure squark pair production. For example at point P2 demanding $P_{T}\left(q_{i}\right)>300 \mathrm{GeV}$, the $\mathrm{KK}$ top at $970 \mathrm{GeV}$ contributes $\Delta \sigma \approx 3.5 \mathrm{fb}$.

We simulate the production of our LSP with WHIZARD, and all following statements refer to hadronic cross sections using the MSTW2008 LO PDF sets from the LHAPDF library [6]. The SM cross section for $p p \rightarrow b \bar{b}, t \bar{t}$ at $\sqrt{s}=14 \mathrm{TeV}$ is very large compared to our signal, at around $\sigma>10^{4} \mathrm{fb}$ for cuts $P_{T}\left(q_{i}\right)>300 \mathrm{GeV}$. One should avoid as much as possible misattributions of such processes, for example by cutting on the angular distance 


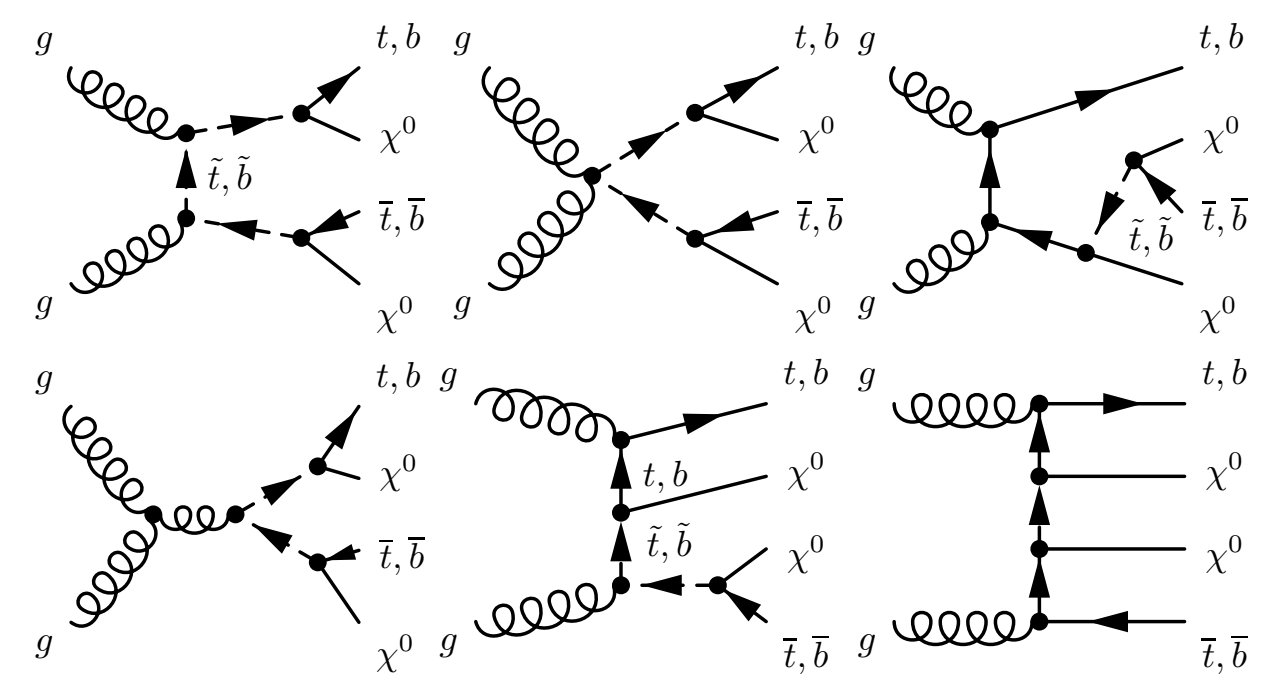

Figure 4: The relevant tree level contributions (up to charge conjugation and crossing) to associated heavy quark and LSP pair production with a $g g$ initial state.

\begin{tabular}{c|c|c|c|c|c} 
Variable & I & II.1 & II.2 & II.3 & II.3' \\
\hline \hline$P_{T}(q), P_{T}(\bar{q})$ & - & $>100 \mathrm{GeV}$ & $>300 \mathrm{GeV}$ & $>100 \mathrm{GeV}$ & $>100 \mathrm{GeV}$ \\
\hline$P_{T}(q, \bar{q})$ & - & - & - & $>300 \mathrm{GeV}$ & $>300 \mathrm{GeV}$ \\
\hline$\Delta \phi(q, \bar{q})$ & - & - & - & {$\left[0,160^{\circ}\right]$} & -
\end{tabular}

Table 4: Definitions of kinematic cuts on visible final state partons. The $q, \bar{q}$ refer to the two quarks in the four parton final state, $\Delta \phi$ denotes the azimuthal angular distance in the lab frame.

between the jets. At the $2 \rightarrow 4$ parton level, the SM background from $p p \rightarrow t \bar{t} \nu \bar{\nu}$ is important, and we want to separate it from our contributions with massive neutralinos. One efficient way to do this is by cutting on the transverse momentum of the quarks. For $P_{T}(q)>300 \mathrm{GeV}$ the cross section is reduced down to $\approx 10 \mathrm{fb}$. The remaining neutrinos are dominantly produced from $Z$ decays.

\subsection{Production of $t \bar{t}+\mathbb{E}_{T}$ from Neutralinos}

The SM background $p p \rightarrow \nu \bar{\nu} t \bar{t}$ is only moderately large. The classes of diagrams contributing to the signal at this order are shown in figure 3 for initial quarks and in figure 4 for initial gluons. In addition to the 
standard cuts $M(q, \bar{q}) \in[10, \infty] ; M($ parton, $q), M($ parton, $\bar{q}) \in[-\infty,-10]$; $E$ (parton) $>20 ; \eta(q), \eta(\bar{q}) \in[-5,5]$ we introduce the set of cuts shown in table 4 to further suppress backgrounds. The cuts on the transverse momentum of the quarks, II.1 and II.2, are efficient in reducing the $2 \rightarrow 4$ parton SM background $p p \rightarrow \nu \bar{\nu} t \bar{t}$. Rather than imposing stronger bounds on the transverse energy of the quarks, we can use the azimuthal distance distribution. We introduce a further set of cuts to exploit the larger contribution of top quarks from LSP production which are not back to back, II.3. Using these cuts, we vary the quark bulk masses according to table 2, and simulate events corresponding to an integrated luminosity of $\int \mathcal{L}=200 \mathrm{fb}^{-1}$ at $\sqrt{s}=14 \mathrm{TeV}$. The results for the final state $\chi^{0} \chi^{0} t \bar{t}$ are shown in figure 5 . While the masses of the lightest $\tilde{t}$ states have the largest influence on the total cross section, the branching ratio of $\tilde{t}$ to LSPs determines how strong the signal will be for this final state alone. The impact of contributions from KK gluons is illustrated by the comparison between points P2 and P3, the latter of which has vanishing lefthanded $q q g^{\prime}$ couplings. We find that cross sections can be reduced dramatically for exact delocalization. The remaining contributions are basically due to the production of onshell stop pairs which entails a typical $P_{T}$ distribution with a cutoff at twice the $\chi$ or $t$ momentum given by the kinematical function,

$$
P_{T}(q \bar{q})<\lambda^{1 / 2}\left(m_{\tilde{t}}^{2}, m_{t}^{2}, m_{\chi}^{2}\right) / m_{\tilde{t}}
$$

which is approximately equal to $m_{\tilde{t}}$ for $m_{\tilde{t}} \gg m_{t} \approx m_{\chi}$.

\subsubsection{Production of $b \bar{b}+\mathbb{E}_{T}$ from Neutralinos}

Essentially the same statements are true for this final state, except that the SM background $p p \rightarrow \bar{\nu} \nu \bar{b} b$ is larger (in particular in the low $\not_{T}$ region), and the signal cross section is smaller due to the large branching fraction of $\Gamma\left(\tilde{b} \rightarrow t \chi^{-}\right)$. Alternatively, one could use cuts I and only consider the region $P_{T}>500 \mathrm{GeV}$, but the resulting distribution has an unremarkable shape which is hard to distinguish from neutrino production. Thus, this channel alone is not well suited for missing energy observations.

\subsection{Production and Decay of the NLSP}

In the previous sections we have considered the leading order contributions to neutralino pair production in association with third generation quarks at the $2 \rightarrow 4$ parton level. There is however another kind of events which might not be easily distinguishable from LSP production in an experiment, namely 

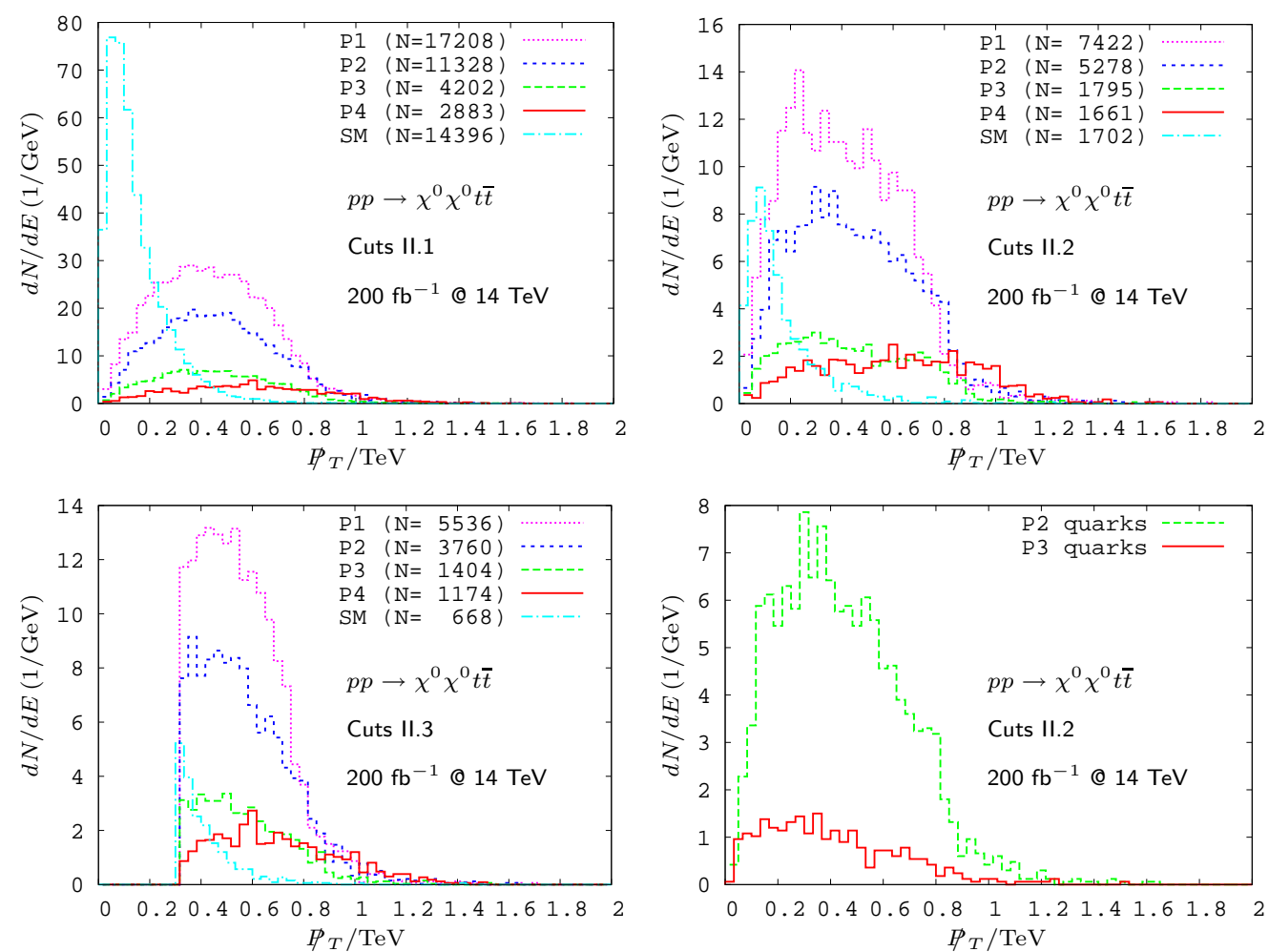

Figure 5: Missing energy from LSP and neutrino production in association with top pairs for different quark localizations and cuts on invariant masses and azimuthal angle (all MSTW08). The line marked SM shows the missing energy in $p p \rightarrow \nu \bar{\nu} t \bar{t}$. For one set of cuts the results for quarks in the initial state are compared for two different localizations. 
the production of chargino NLSPs or of one chargino NLSP and a neutralino LSP. Also, there are regions in parameter space where

$$
\Gamma\left(\tilde{q} \rightarrow \chi^{ \pm} q\right) \gg \Gamma\left(\tilde{q} \rightarrow \chi^{0} q\right)
$$

for the light third generation squarks (in particular $\tilde{b}$ ), making NLSP production and decay the dominant source of missing energy. Our charginos are close to the lower experimental exclusion bound and decay via $\chi^{+} \rightarrow$ $\chi^{0} W^{+*} \rightarrow \chi^{0} f_{u} \bar{f}_{d}$. They are very narrow $\left(\Gamma \approx 10^{-7} \mathrm{GeV}\right)$ due to the offshell intermediate $W$, yet unlike in some models with light gravitino LSP, the corresponding lifetime is still negligible. The fermion pair has an energy of $\approx 10 \ldots 20 \mathrm{GeV}$ in the chargino center of mass frame. Depending on how well such processes can be resolved in the experiments, this will be an important channel for the observation of LSP or NLSP production. In the detector, one might see missing energy with one lepton or comparatively soft jets (few tens of $\mathrm{GeVs}$ ) and two $b$ or $t$ jets, so chargino production events would look similar to the signal discussed above. We therefore close this section by studying the processes

$$
p p \rightarrow \chi^{+} \chi^{-} t \bar{t}, \chi^{+} \chi^{-} b \bar{b}, \quad p p \rightarrow \chi^{0} \chi^{-} t \bar{b}, \quad p p \rightarrow \chi^{0} \chi^{+} \bar{t} b
$$

to which the three point couplings $W^{+, n} \chi^{-} \chi^{0}$ and $Z^{n} \chi^{+} \chi^{-}$and for the latter also $W W Z$ can make important contributions, in contrast to neutralino pair production. Since the results below are for vanishing IR kinetic terms (and thus very heavy $W$ and $Z$ resonances), these contributions can be both enhanced due to phase space and decreased further above the energy scale at which unitarization sets in. In the case of final states with charginos, the $P_{T}$ of the $b$ and $t$ jets is not exactly equal to $P_{T}$ any more, but only up to the $P_{T}$ of the softer jet(s) from the chargino decay products (assuming $t, b$ decay into visible particles only). The distribution of $P_{T}(q \bar{q})$ and chargino boosts in the lab frame is shown in figure 6 for chargino pairs and in figure 7 for mixed chargino and neutralino production. The boost distribution of the charginos can be used to infer how hard the jets or leptons from the chargino decay products are going to be. The maximum is at around $\gamma\left(\chi^{ \pm}\right)=E_{\chi} / m_{\chi} \approx 3 \ldots 5$ depending on the parameters, leading to rather soft jets, but a significant number of events can reach $\gamma\left(\chi^{ \pm}\right)>5$. These cross sections are even larger than those for pure neutralino production, but it is subject of further study how well this type of events can be distinguished at the LHC. The proper treatment of the background to this type of processes would require production of 8 partons in the final state to account for the three body decays of two $\chi^{ \pm} \mathrm{s}$ via offshell $W \mathrm{~s}$, and possibly detector simulations which are beyond the scope of this work. In figure 8 , we compare 

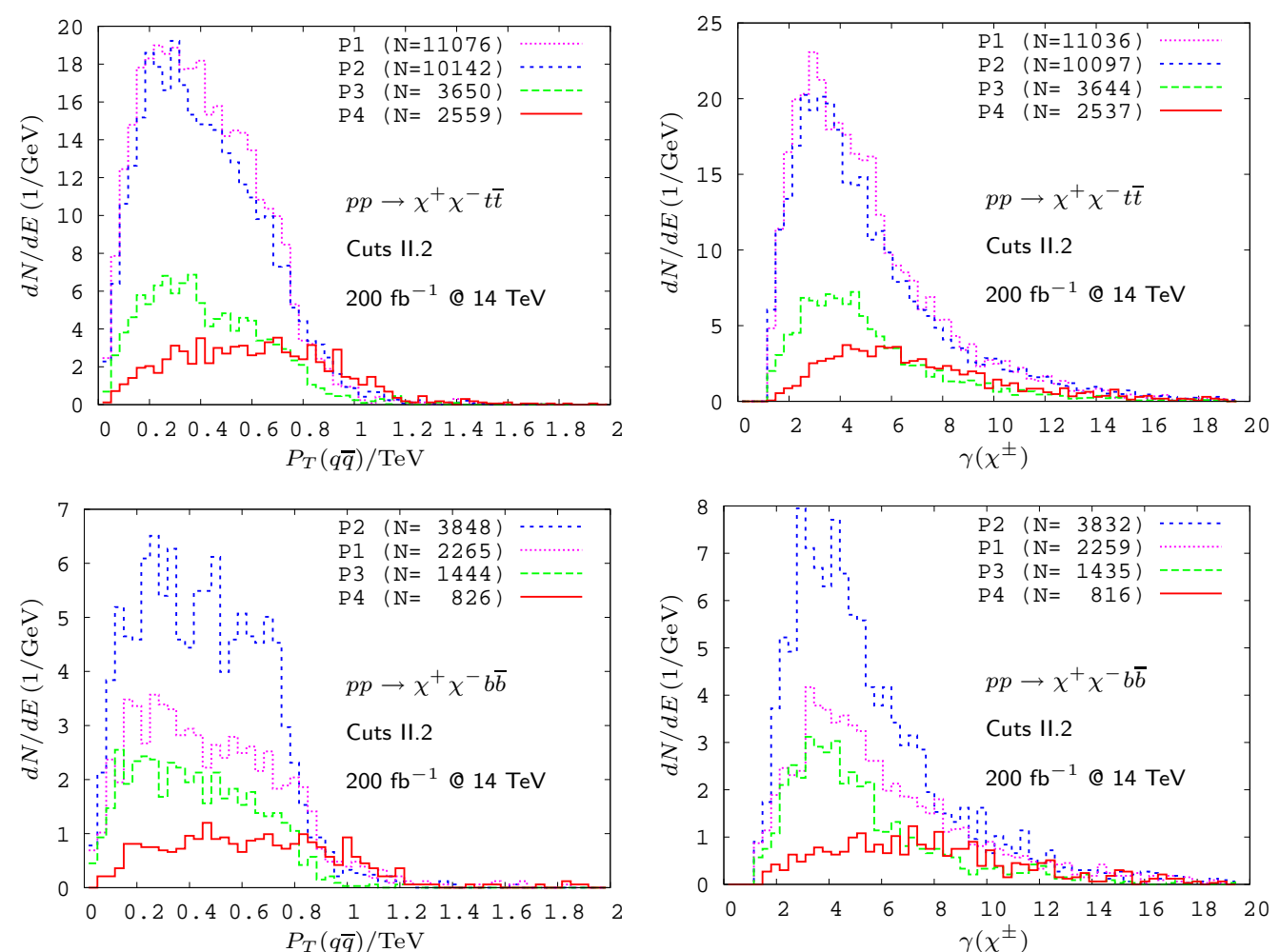

Figure 6: Total transverse momenta and boosts of charginos produced in association with top and bottom pairs for different quark localizations after cuts II.2 (all MSTW08). The total $P_{T}$ is shown as an approximation to $P_{T}$ which will have further contributions from the decay products.

the various final states for two different sets of third generation localization in which the bulk masses of the $S U(2)_{L}$ and $S U(2)_{R}$ charged hypermultiplets are exchanged. In any case, NLSP production gives us an equally important source of missing energy.

\subsection{Backgrounds from Leptonic t Decays}

In the detector, the final states $\chi^{0} \chi^{0} b \bar{b} q \bar{q} l \bar{\nu}, \chi^{0} \chi^{0} b \bar{b} q \bar{q} \nu \bar{l}$ or $\chi^{0} \chi^{0} b \bar{b} l \bar{\nu} \nu \bar{l}$ as they appear in the processes discussed above, if one or both tops decay leptonically, and $b \bar{b} q \bar{q} l \bar{\nu}, b \bar{b} q \bar{q} \nu \bar{l}$ or $b \bar{b} l \bar{\nu} \nu \bar{l}$ (e.g. from top pair production) are not distinguishable except for their kinematics, and all produce missing energy signatures. Both groups can arise from top decays and thus show the typical edge in the $\bar{l} b$ or $\bar{b} l$ invariant mass, but while the angle in the center 

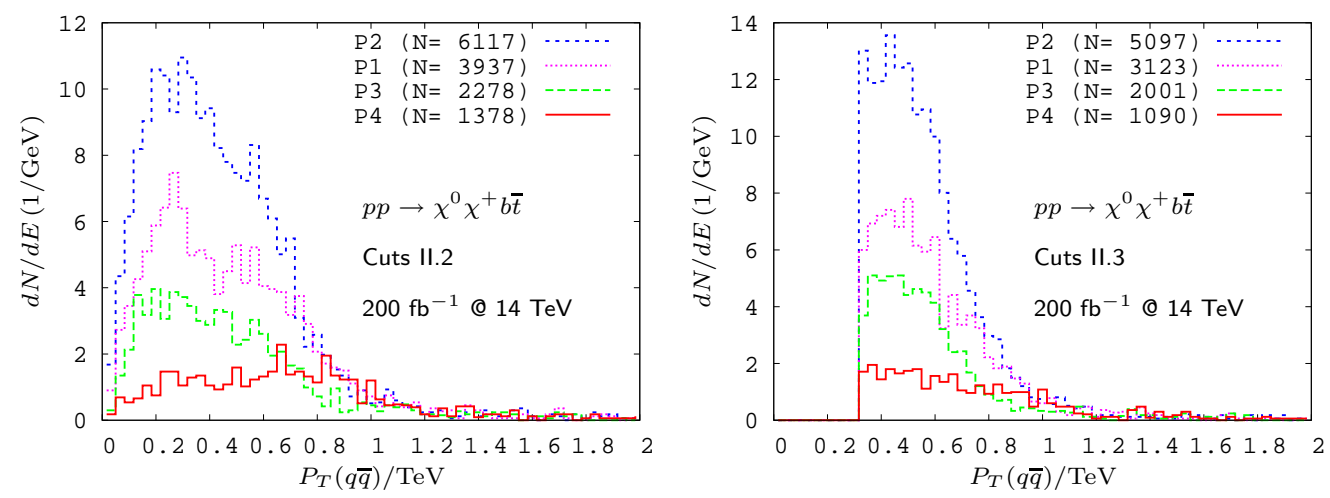

Figure 7: Total transverse momenta of electroweak gauginos produced in association with $b \bar{t}$ for different quark localizations after cuts II.2 and II.3 (all MSTW08). The total $P_{T}$ is shown as an approximation to $\not_{T}$ which will have further contributions from the decay products.

of mass between the tops is close to $180^{\circ}$ in the case of pair production, the neutralinos in the signal process may produce substantial recoil. One might hope that cuts on the azimuthal angle such as cuts II.3 favor the signal contribution. To check this we implement the cuts II.2 and II.3 for the visible decay products, $\Delta \phi(t, \bar{t}) \rightarrow \Delta \phi\left(b l^{+}, \bar{b} l^{-}\right)$and $p_{T}(t) \rightarrow p_{T}\left(b l^{+}\right)$in the SM process $p p \rightarrow b \bar{b} l^{+} l^{-} \nu \bar{\nu}$. In addition, we introduce a separation cut $\sqrt{\Delta \phi^{2}+\Delta \eta^{2}}>0.3$ for the two $b$ quarks which reduces the SM background from initial quarks by half. The results are shown in figure 9. Apparently, the angular cut in combination with $P_{T}$ cuts is indeed efficient, reducing the missing energy background from leptonic decays down to $\approx 8 \mathrm{fb}$. However, when comparing the signal contributions to this, one has to consider that only a fraction of the top pairs decay purely leptonically.

\section{Conclusions}

We have given a short overview over our supersymmetric warped model and an application to the EWSB sector of higgsless models as introduced in [1]. Motivated by the presence of a realistic CDM candidate and naturally light third generation squark resonances in this model, we have simulated the production of the LSP and NLSP in association with third generation quarks with respect to their missing energy signatures using an implementation in WHIZARD. Indeed, the production of the lightest $\tilde{t}$ and $\tilde{b}$ resonances is the most important factor in this class of processes at the $2 \rightarrow 4$ parton level. 

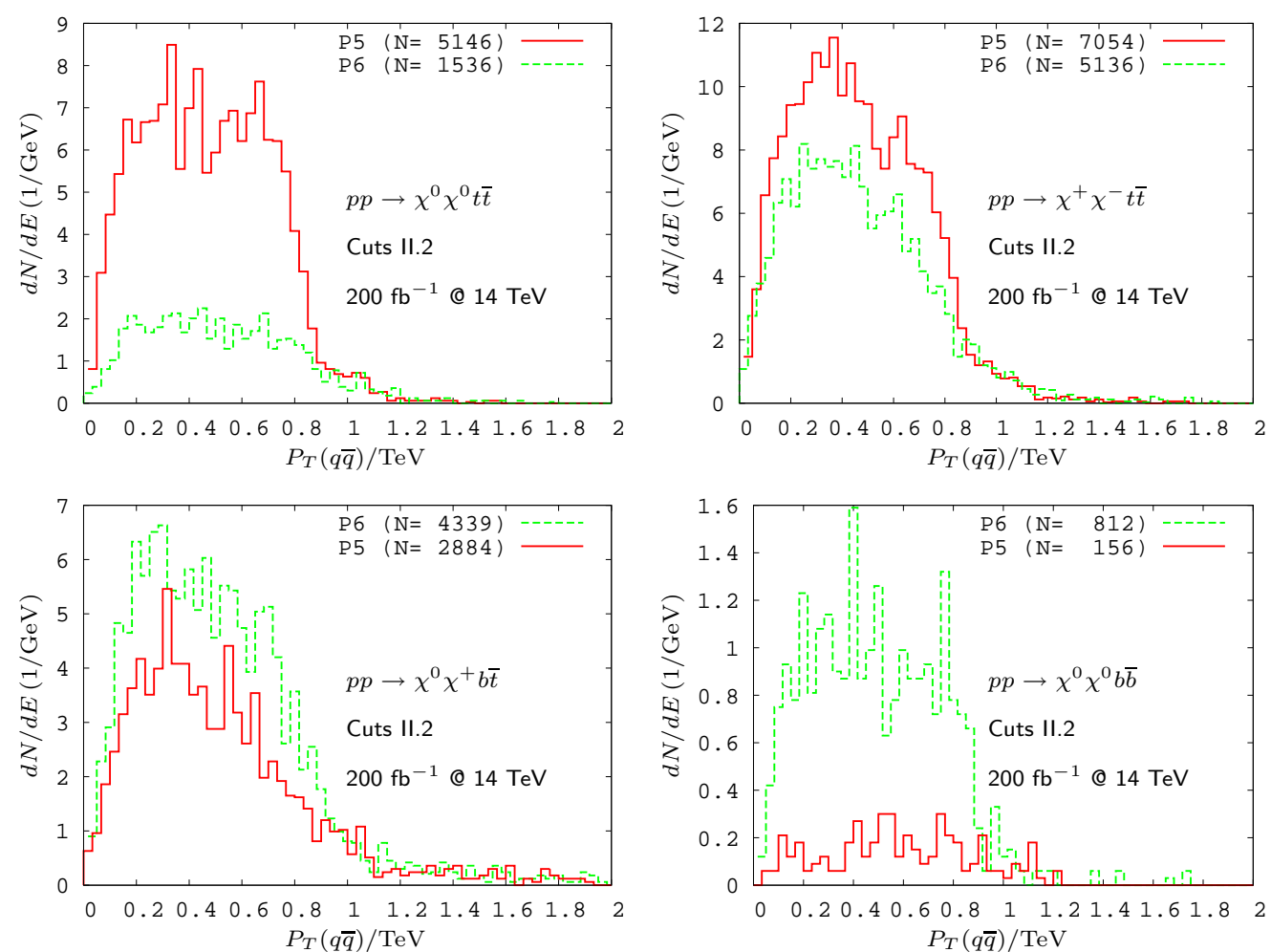

Figure 8: Missing energy from LSP and NLSP production for different third generation bulk masses. The results from P5 and P6 are compared using cuts II.2. (all MSTW08)
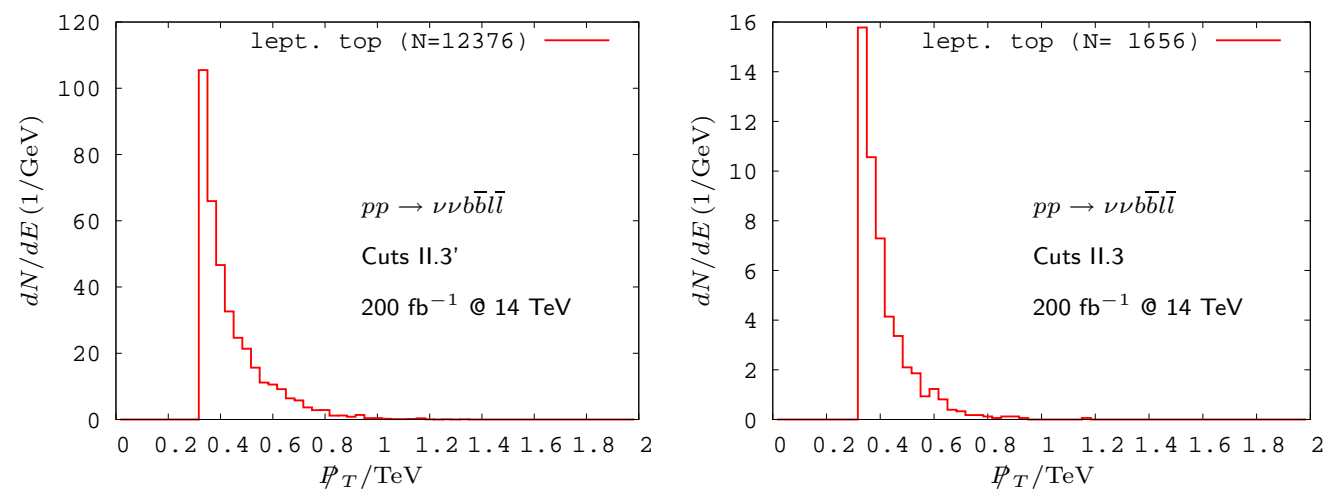

Figure 9: Missing energy in $p p \rightarrow \nu \nu b \bar{b} l \bar{l}$ (predominantly) from leptonic top decays in the SM (all MSTW08). The modified cuts II.3' are II.3 without the cut on the azimuthal angular distance to illustrate its efficiency. 
Consequently their mass, which depends on the third generation hypermultiplet bulk mass parameters $c_{L 3}$ and $c_{R 3}$, determines the order of magnitude of cross sections. Since the NLSPs are very light in our model, the final states involving $\chi^{0}$ and $\chi^{ \pm}$are equally important, the relative strength in each case being determined by the branching ratios of $\tilde{b}$ and $\tilde{t}$ decays. While the decay products of the NLSP should in principle be observable as jets or leptons with energies below $\approx 100 \mathrm{GeV}$ depending on the mass splitting $\Delta m(\chi)$, we have used for simplicity the transverse momentum of the NLSPs as an approximation to $\mathbb{P}_{T}$.

Taking into consideration the leading SM background at the $2 \rightarrow 4$ parton level, $p p \rightarrow \bar{\nu} \nu \bar{q} q$, we find that cuts on the transverse momenta of final state quarks are an efficient means of increasing the signal to background ratio. Depending on the particular decay mode of the tops one is interested in, missing energy from the resulting neutrinos can become the dominant SM background. We have illustrated this with a short analysis of the purely leptonic decay mode of SM top pair production, and have demonstrated how this contribution can be reduced using $P_{T}$ and angular cuts. Again, this background drops quickly at large $\mathbb{P}_{T}$, and allows us to observe LSP production in the region $P_{T} \gtrsim 400 \mathrm{GeV}$.

The strength of the signal strongly depends on the quark localization parameters both in the first and third generation. For the following results we have assumed the cuts II.2, restricting the quark transverse momenta to $P_{T}(q)>300 \mathrm{GeV}$. For our benchmark points P1, P2 featuring one rather light $\tilde{b}$ and $\tilde{t}$ resonance, we find a signal cross section of $\sigma_{s} \approx 130 \mathrm{fb}$ for the combination of all final states involving $\chi^{0 \pm} \chi^{0 \mp}$ at $14 \mathrm{TeV}$. The two points yield essentially the same total cross section and differ only in the relative importance of the various final states. It drops to $\sigma_{s} \approx 60 \mathrm{fb}$ at $14 \mathrm{TeV}$ for P3 with delocalized light quarks. At P4, which features IR localized third generation hypermultiplets, the KK mode splitting is smaller leading to heavy squarks. The resulting cross section including all final states is $\sigma_{s} \approx 40 \mathrm{fb}$ at $14 \mathrm{TeV}$. Worse prospects are imaginable for more extreme IR localization in combination with delocalized light quarks.

The production of neutrinos in association with third generation quark pairs contributes a background cross section of $\sigma_{b} \approx 20 \mathrm{fb}$ at $14 \mathrm{TeV}$, but mainly at missing momenta $P_{T}<300 \mathrm{GeV}$ which is significantly below the cutoff of $\sigma_{s}$ at $P_{T} \approx \lambda^{1 / 2}\left(m_{\tilde{q}}^{2}, m_{q}^{2}, m_{\chi}^{2}\right) / m_{\tilde{q}}$ for most of the parameter space. In our discussion we have not considered more complex backgrounds, detector effects or misattributions of final states in detail. However we argue that one can apply the discussions from the literature on $\tilde{t}$ and $\tilde{b}$ production (e.g. [7]) to our case, keeping in mind that additional contributions from the KK sector in warped models can enhance signal cross sections significantly. 
The production of heavy intermediates with $m \gtrsim 1 \mathrm{TeV}$ suffers severely from reduced parton densities in the large $x$ region at center of mass energies below $\sqrt{s}=10 \mathrm{TeV}$ as they are scheduled for the first run of the LHC. For example, after cuts II.2 and P2, the cross section $\sigma\left(p p \rightarrow \chi^{0} \chi^{0} t \bar{t}\right)$ drops by a factor of $\approx 4$. Fortunately, the same is true for SM neutrino production, thanks to the strong $P_{T}$ cut.

\section{Acknowledgments}

We thank Thomas Trefzger for useful discussions on the ATLAS detector. This research is supported by Deutsche Forschungsgemeinschaft through the Research Training Group 1147 Theoretical Astrophysics and Particle Physics, and by Bundesministerium für Bildung und Forschung Germany, grants 05HT6WWA and 05H09WWE. A. K. is also supported by Deutsche Forschungsgemeinschaft through grant RE 2850/1-1.

\section{References}

[1] A. Knochel and T. Ohl, Phys. Rev. D 78, 045016 (2008) arXiv:0805.1379 [hep-ph]].

[2] M. Moretti, T. Ohl and J. Reuter, arXiv:hep-ph/0102195; W. Kilian, LC-TOOL-2001-039; W. Kilian, T. Ohl and J. Reuter, arXiv:0708.4233 [hep-ph]].

[3] C. Csaki, C. Grojean, L. Pilo, and J. Terning, Phys. Rev. Lett. 92, 101802 (2004), arXiv:hep-ph/0308038; C. Csaki, C. Grojean, H. Murayama, L. Pilo, and J. Terning, Phys. Rev. D69, 055006 (2004), arXiv:hep-ph/0305237]; C. Csaki, C. Grojean, J. Hubisz, Y. Shirman, and J. Terning, Phys. Rev. D70, 015012 (2004), arXiv:hep-ph/0310355]; G. Cacciapaglia, C. Csaki, C. Grojean, and J. Terning, Phys. Rev. D70, 075014 (2004), arXiv:hep-ph/0401160; G. Cacciapaglia, C. Csaki, C. Grojean, and J. Terning, Phys. Rev. D71, 035015 (2005), arXiv:hep-ph/0409126].

[4] N. Marcus, A. Sagnotti and W. Siegel, Nucl. Phys. B 224, 159 (1983); N. Arkani-Hamed, T. Gregoire, and J. G. Wacker, JHEP 03, 055 (2002), arXiv:hep-th/0101233]; D. Marti and A. Pomarol, Phys. Rev. D64, 105025 (2001), |arXiv:hep-th/0106256]. 
[5] G. Cacciapaglia, C. Csaki, G. Marandella, and J. Terning, Phys. Rev. D75, 015003 (2007), arXiv:hep-ph/0607146].

[6] M. R. Whalley, D. Bourilkov and R. C. Group, arXiv:hep-ph/0508110.

[7] T. Han, R. Mahbubani, D. G. E. Walker and L. T. E. Wang, JHEP 0905, 117 (2009) arXiv:0803.3820 [hep-ph]]. 